\title{
INFLUÊNCIA DA CAPACIDADE PARA O TRABALHO NA QUALIDADE DE VIDA DE BOMBEIROS MILITARES*
}

\author{
Bruno Gonçalves de Oliveira ${ }^{1}$, Eliane dos Santos Bomfim ${ }^{1}$, Ícaro José Santos Ribeiro ${ }^{1}$, Paulo Henrique \\ Ribeiro Fernandes Almeida ${ }^{2}$, Rita Narriman Silva de Oliveira Boery ${ }^{3}$, Eduardo Nagib Boery ${ }^{4}$
}

\begin{abstract}
RESUMO: Objetivo: avaliar a influência da capacidade para o trabalho na qualidade de vida de bombeiros militares. Método: estudo censitário de base populacional do tipo transversal, realizado de janeiro a março de 2015, com 101 bombeiros do Grupamento de Bombeiros Militares de municípios da região Sul e Sudoeste da Bahia. Foram utilizados no estudo instrumentos autoaplicáveis para avaliar as características sociodemográficas e laborais, qualidade de vida e capacidade para o trabalho. Resultado: constatou-se que os bombeiros militares com menor ou igual a cinco anos de serviço obtiveram melhores percepções de qualidade de vida nos domínios físico e relações sociais. Quanto à capacidade para o trabalho, observou-se que os trabalhadores com boa e ótima capacidade obtiveram melhores percepções nos domínios físico, psicológico, social e meio ambiente. Conclusão: os resultados podem auxiliar nas intervenções e melhorias nas condições de trabalho de profissionais de resgate como bombeiros, enfermeiros e médicos.
\end{abstract}

DESCRITORES: Bombeiros; Condições de trabalho; Qualidade de vida; Saúde do trabalhador.

\section{INFLUENCE OF WORK ABILITY ON THE QUALITY OF LIFE OF MILITARY FIREFIGHTERS}

\begin{abstract}
Objective: to evaluate the influence of the work ability on the quality of life of military firefighters. Method: a crosssectional, population-based, census study, conducted from January to March 2015, with 101 firefighters from the Military Fire Brigade Group of municipalities in the South and Southwest of Bahia. Self-report instruments were used in the study to evaluate the sociodemographic and work characteristics, quality of life and work ability. Results: it was found that military firefighters with five years or less of service obtained better perceptions of quality of life in the physical and social relationships domains. Regarding the work ability, it was observed that workers with good and very good ability obtained better perceptions in the physical, psychological, social relationships and environment domains. Conclusion: the results can support interventions and improvements in the working conditions of rescue professionals such as firefighters, nurses and physicians.
\end{abstract}

DESCRIPTORS: Firefighters; Work conditions; Quality of life; Worker's health.

\section{INFLUENCIA DE LA CAPACIDAD LABORAL EN LA CALIDAD DE VIDA DE BOMBEROS MILITARES}

RESUMEN: Objetivo: Evaluar la influencia de la capacidad laboral en la calidad de vida de bomberos militares. Método: Estudio censario de base poblacional, tipo transversal, realizado de enero a marzo de 2015 con 101 bomberos del Cuartel de Bomberos Militares de municipios del sur y sureste de Bahia. Fueron utilizados instrumentos autoaplicables para evaluar características sociodemográficas y laborales, calidad de vida y capacidad laboral. Resultado: Se constató que los bomberos militares con cinco años o menos de servicio mostraron mejores percepciones de calidad de vida en los dominios físico y relaciones sociales. Respecto a la capacidad laboral, se observó que los trabajadores con buena y óptima capacidad mostraron mejores percepciones en los dominios físico, psicológico, social y medio ambiente. Conclusión: Los resultados pueden colaborar en intervenciones y mejoras de las condiciones de trabajo de los profesionales de rescate, como bomberos, enfermeros y médicos.

DESCRIPTORES: Bomberos; Condiciones de Trabajo; Calidad de Vida; Salud Laboral. *Artigo extraído da dissertação de mestrado "Fatores associados à qualidade de vida de bombeiros militares". Universidade
Estadual do Sudoeste da Bahia, 2015.

${ }^{1}$ Enfermeiro. Doutorando em Ciências da Saúde. Universidade Estadual do Sudoeste da Bahia. Jequié, BA, Brasil.

${ }^{2}$ Farmacêutico. Doutorando em Farmácia. Universidade Federal de Minas Gerais. Belo Horizonte, MG, Brasil.

${ }^{3}$ Enfermeira. Pós- Doutora em Enfermagem. Docente de Pós-Graduação em Enfermagem da Universidade Estadual do Sudoeste da Bahia. Jequié, BA, Brasil.

${ }^{4}$ Enfermeiro. Doutor em Enfermagem. Docente de Pós-Graduação em Enfermagem da Universidade Estadual do Sudoeste da Bahia. Jequié, BA, Brasil. 


\section{INTRODUÇÃO}

Os Bombeiros Militares (BM) constituem uma das categorias profissionais que mais enfrentam condições adversas no trabalho, ao executar serviços que exigem grande esforço físico, além de precisão e ação rápida durante as ocorrências, sendo que qualquer deslize pode colocá-lo em risco de morte $^{(1)}$. Essas situações podem proporcionar desgaste tanto físico como mental para o profissional ${ }^{(2)}$. Por esta razão, o estudo da sua Qualidade de Vida (QV) vem despertando interesse, no sentido de buscar melhorias nas condições laborais, no ambiente psicossocial, na promoção da saúde e no desenvolvimento da competência de cada profissional no contexto de trabalho ${ }^{(3-4)}$.

Ressalta-se que a QV é um conceito subjetivo, multidimensional, que envolve elementos de avaliação tanto positiva como negativa, e estabelece relação entre diferentes domínios (físico, psicológico, nível de independência, social e ambiental) ${ }^{(5-6)}$. Pelo caráter multidimensional, a QV pode repercutir de diversas formas nos aspectos laborais dos indivíduos, desde aspectos relacionados à promoção da saúde até o adoecimento pelas doenças relacionadas ao labor, aspectos fundamentais para o mantimento da sua capacidade de trabalho ${ }^{(7)}$.

A capacidade para o trabalho do BM pode ser influenciada pelo seu estado de saúde, além disso, as interações estabelecidas com estas podem proporcionar aumento de sintomas como o estresse, ansiedade, mudanças na qualidade do sono, transtornos mentais, além do desenvolvimento de doenças psicossomáticas e cardiovasculares ${ }^{(8-9)}$.

O BM submete-se a condições de exposição de riscos no trabalho, quer seja pelo contato com agentes físicos e biológicos durante as ocorrências, ou pelas longas jornadas de trabalho, que ocasionam o cansaço e sobrecarga no serviço, afetando o desempenho e a rotina laborais, diminuindo consequentemente sua capacidade para o trabalho ${ }^{(10)}$.

Diante do exposto, este estudo tem como finalidade contribuir para a melhoria da QV do BM, além de incentivar (re)organização dos serviços de saúde tanto estrutural como funcional, no intuito de oferecer ações de promoção à saúde a este grupo de trabalhadores. Objetiva-se avaliar a influência da capacidade para o trabalho na qualidade de vida de bombeiros militares.

\section{MÉTODO}

Trata-se de um estudo epidemiológico, de base populacional de caráter transversal, realizado no Grupamento de Bombeiros Militares (GBM) de municípios da região Sul e Sudoeste da Bahia, Brasil, no período de janeiro a março de 2015. O $8^{\circ} \mathrm{GBM}$ é composto por 128 bombeiros que atuam no serviço operacional e administrativo em 24 municípios da região Sul e Sudoeste da Bahia.

Foram incluídos no estudo BM de ambos os sexos que exerciam serviços operacionais e que após esclarecimento sobre os objetivos do estudo, concordarem em participar, assinando o Termo de Consentimento livre e esclarecido (TCLE). Foram excluídos 25 bombeiros que realizavam atividades administrativas e houve a perda de dois que se encontravam de férias. Assim a amostra foi constituída de 101 bombeiros.

A aplicação dos instrumentos foi acompanhada por pesquisadores treinados e instruídos, a fim de evitar possíveis vieses. Foi utilizado formulário constituído por três blocos temáticos: características sociodemográficas e do trabalho, inquérito sobre a QV e inquérito sobre a capacidade para o trabalho.

As variáveis sociodemográficas avaliadas foram: sexo, faixa etária, situação conjugal, escolaridade, raça/cor ${ }^{(11)}$ e renda mensal como bombeiro. Com relação às características laborais, foram verificadas as seguintes variáveis: outros vínculos empregatícios, posto hierárquico, punição no serviço, satisfação no ambiente de trabalho e situações de emergência. Estando postulado que o tempo de serviço pode afetar a capacidade de trabalho dos indivíduos ${ }^{(7)}$, optou-se por apresentar a caracterização da população estudada de acordo com tal variável categorizada aqui como menor ou igual a cinco anos $(\leq 5)$ ou maior que cinco anos (>5).

A QV foi avaliada por meio do instrumento World Health Organization Quality of Life (WHOQOL- 
Bref) que foi validado para o uso no Brasil ${ }^{(6)}$. O instrumento é constituído por 24 questões agrupadas em quatro domínios:

- Físico: 3. Dor e desconforto; 4. Energia e fadiga; 10. Sono e repouso; 15. Mobilidade; 16. Atividades da vida cotidiana; 17. Dependência de medicação ou de tratamentos; 18. Capacidade de trabalho.

- Psicológico: 5. Sentimentos positivos; 6. Pensar, aprender, memória e concentração; 7. Autoestima; 11. Imagem corporal e aparência; 19. Sentimentos negativos; 26. Espiritualidade/religião/ crenças pessoais

- $\quad$ Relações sociais: 20. Relações pessoais; 21. Suporte (Apoio) social; 22. Atividade sexual

- Meio ambiente: 8. Segurança física e proteção; 9. Ambiente no lar; 12. Recursos financeiros; 13. Cuidados de saúde e sociais: disponibilidade e qualidade; 14. Oportunidades de adquirir novas informações e habilidades; 23. Participação em, e oportunidades de recreação/lazer; 24. Ambiente físico: (poluição/ruído/trânsito/clima); 25. Transporte.

- Duas questões que avaliam a QV de modo geral e a satisfação com a própria saúde ${ }^{(6)}$.

Para avaliar a capacidade para o trabalho, foi utilizado o Índice de Capacidade para o Trabalho (ICT), traduzido e validado no Brasil ${ }^{(12)}$. Esse instrumento fornece um escore que varia de sete (pior índice) a 49 pontos (melhor índice), os quais foram categorizados em quatro níveis: baixo (7-27), moderado (2836), bom (37-43) e ótimo (44-49).

A análise dos dados foi processada pelo programa Statistical Package for the Social Sciences (SPSS) 21.0. Para fins de constatação do padrão de distribuição dos dados, foi aplicado o teste KolmogorovSmirnov, sendo evidenciada a não normalidade $(\mathrm{p}<0,05)$.

As variáveis qualitativas foram descritas através de frequências absolutas e relativas, enquanto as quantitativas em mediana e intervalo interquartílico. Objetivando a constatação de diferenças entre os domínios da QV e o tempo de serviços dos BM, foi realizado o teste de Mann Whitney. No mesmo sentido, para a comparação dos domínios da QV com as categorias do ICT foi realizado o teste de Kruskal-Wallis. O nível de significância adotado nos testes foi de $p<0,05$. Os resultados foram apresentados em mediana e intervalo interquartil.

O estudo foi aprovado pelo Comitê de Ética em Pesquisa (CEP) da Universidade Estadual do Sudoeste da Bahia, de acordo à Resolução do Conselho Nacional de Saúde n ${ }^{\circ} 466 / 2012^{(13)}$, sob parecer no 972.480.

\section{- RESULTADOS}

Entre os avaliados, a mediana de idade foi de 39 anos (34 - 43,5), variando de 24 a 51 anos. Houve a predominância de indivíduos com mais de 5 anos de serviço, $76,2 \%(n=77)$, sendo $81,8 \%$ ( $n=63)$ do sexo masculino e $52 \%(n=40)$ com idade acima de 40 anos e com ensino médio.

Quanto às características laborais, evidenciou-se que $94,8 \%(n=73)$ estavam satisfeitos com o trabalho, $79,3(n=61)$ nunca receberam punição, $46,7 \%(n=36)$ ocupavam o posto hierárquico de soldados e $58,4 \%$ não vivenciaram momento traumático durante o trabalho.

Na Tabela 1 destacam-se as características sociodemográficas e laborais dos BM, segundo o tempo de serviço (anos) na profissão. 
Tabela 1 - Características sociodemográficas e laborais dos bombeiros estratificadas pelo tempo de serviço (anos) no trabalho. Jequié, BA, Brasil, 2015

\begin{tabular}{|c|c|c|c|c|}
\hline \multirow[t]{3}{*}{ Variáveis } & \multicolumn{4}{|c|}{ Tempo de serviço como Bombeiro } \\
\hline & \multicolumn{2}{|c|}{$\leq \mathbf{5}$ anos } & \multicolumn{2}{|c|}{$>5$ anos } \\
\hline & n (24) & $\%$ & n (77) & $\%$ \\
\hline \multicolumn{5}{|c|}{ CARACTERÍSTICAS SOCIODEMOGRÁFICAS } \\
\hline \multicolumn{5}{|c|}{ Sexo } \\
\hline Masculino & 20 & 83,3 & 63 & 81,8 \\
\hline Feminino & 4 & 16,7 & 14 & 18,2 \\
\hline \multicolumn{5}{|l|}{ Faixa etária (em anos) } \\
\hline $21 \mathrm{H} 30$ & 15 & 62,5 & - & - \\
\hline $31 \mathrm{H} 40$ & 9 & 37,5 & 37 & 48 \\
\hline$>40$ & - & - & 40 & 52 \\
\hline \multicolumn{5}{|l|}{ Situação conjugal } \\
\hline Com companheiro(a) & 12 & 50 & 57 & 74 \\
\hline Sem companheiro(a) & 12 & 50 & 20 & 26 \\
\hline \multicolumn{5}{|l|}{ Escolaridade } \\
\hline Ensino Médio & 5 & 20,8 & 34 & 44,1 \\
\hline Ensino Superior & 17 & 70,8 & 32 & 41,6 \\
\hline Pós-graduação/Mestrado/Doutorado & 2 & 8,4 & 11 & 14,3 \\
\hline \multicolumn{5}{|l|}{ Raça/Cor } \\
\hline Negros & 18 & 75 & 66 & 85,7 \\
\hline Não Negros & 6 & 25 & 11 & 14,3 \\
\hline \multicolumn{5}{|l|}{ Renda } \\
\hline 3 H 4 salários & 17 & 70,8 & 58 & 75,3 \\
\hline 4 H 5 salários & 3 & 12,5 & 9 & 11,7 \\
\hline 5 salários ou mais & 4 & 16,7 & 10 & 13 \\
\hline \multicolumn{5}{|l|}{ CARACTERÍSTICAS LABORAIS } \\
\hline \multicolumn{5}{|l|}{ Satisfação com o ambiente de trabalho } \\
\hline Sim & - & & 73 & 94,8 \\
\hline Não & 24 & 100 & 4 & 5,2 \\
\hline \multicolumn{5}{|l|}{ Punição no trabalho } \\
\hline Sim & - & - & 16 & 20,7 \\
\hline Não & 24 & 100 & 61 & 79,3 \\
\hline \multicolumn{5}{|l|}{ Posto hierárquico } \\
\hline Soldado & 19 & 79,1 & 36 & 46,7 \\
\hline Cabo & - & - & 18 & 23,4 \\
\hline Sargento & - & - & 16 & 20,8 \\
\hline Oficial & 5 & 20,9 & 7 & 9,1 \\
\hline \multicolumn{5}{|l|}{ Evento traumático } \\
\hline Sim & 5 & 20.9 & 32 & 41,6 \\
\hline Não & 19 & 79,1 & 45 & 58,4 \\
\hline
\end{tabular}

No que concerne à comparação entre os domínios de QV segundo o tempo de serviço na profissão, foi possível evidenciar diferenças estatisticamente significativas. Os BM com tempo de serviço $\leq 5$ anos apresentaram uma melhor percepção de QV nos domínios físico e social quando comparados com o tempo de serviço $>5$ anos (Tabela 2). 
Tabela 2 - Mediana e intervalo interquartil (IQ) dos domínios da qualidade de vida, estratificados pelo tempo de trabalho (anos) como bombeiro $(\mathrm{N}=101)$. Jequié, BA, Brasil, 2015

\begin{tabular}{cccc} 
Domínios do Whoqol-Bref & Tempo de Serviço & Mediana (IQ) & Valor de p \\
\cline { 2 - 4 } Físico & $\leq 5$ & $80,4(71,4-84,8)$ & $0,049^{*}$ \\
\cline { 2 - 4 } & $>5$ & $71,4(62,5-85,7)$ & \multirow{2}{*}{0,273} \\
\cline { 2 - 4 } Psicológico & $\leq 5$ & $81,2(70,8-87,5)$ \\
\cline { 2 - 4 } & $>5$ & $75(70,8-83,3)$ & \multirow{2}{*}{$0,029^{*}$} \\
\cline { 2 - 4 } Relações Sociais & $\leq 5$ & $83,3(75,0-91,6)$ & \multirow{2}{*}{0,329} \\
\cline { 2 - 4 } Meio Ambiente & $>5$ & $75(75,0-83,3)$ & \\
\cline { 2 - 4 } & $>5$ & $65,6(53,1-74,2)$ & 0,643
\end{tabular}

Quanto aos resultados da classificação da capacidade para o trabalho segundo o escore global ICT entre os BM, constatou-se que 5,9\% ( $n=$ seis) apresentavam capacidade baixa, $34,7 \%(n=35)$ moderada, $43,6 \%(n=44)$ boa e $15,8 \%(n=16)$ ótima.

Comparando-se os domínios da QV com as categorias da capacidade para o trabalho, foi evidenciado que os indivíduos com boa capacidade apresentaram melhor percepção de QV no domínio físico. Os indivíduos com capacidade ótima apresentaram melhor percepção de QV nos domínios psicológico, relações sociais e meio ambiente (Tabela 3 ).

Tabela 3 - Comparação dos domínios da qualidade de vida com as categorias estratificadas do ICT dos bombeiros ( $N=101)$. Jequié, BA, Brasil, 2015

\begin{tabular}{|c|c|c|c|}
\hline Domínios do Whoqol-Bref & Capacidade & Mediana (IQ) & $\mathbf{P}$ \\
\hline \multirow{4}{*}{ Físico } & Baixa & $53,5(45,5-65,1)$ & \multirow{4}{*}{$0,006^{*}$} \\
\hline & Moderada & $71,4(60,7-82,1)$ & \\
\hline & Boa & $78,5(67,8-84,8)$ & \\
\hline & Ótima & $73,2(65,1-85,7)$ & \\
\hline \multirow{4}{*}{ Psicológico } & Baixa & $60,1(58,3-68,7)$ & \multirow{4}{*}{$0,017^{*}$} \\
\hline & Moderada & $79,1(70,8-87,5)$ & \\
\hline & Boa & $79,1(70,8-87,5)$ & \\
\hline & Ótima & $81,2(75-90,6)$ & \\
\hline \multirow{4}{*}{ Relações sociais } & Baixa & $70,8(50-75)$ & \multirow{4}{*}{$0,001^{*}$} \\
\hline & Moderada & $75,0(66,6-83,3)$ & \\
\hline & Boa & $75,0(75-83,3)$ & \\
\hline & Ótima & $87,5(77,1-100)$ & \\
\hline \multirow{4}{*}{ Meio ambiente } & Baixa & $51,6(43,7-57)$ & \multirow{4}{*}{$0,038^{*}$} \\
\hline & Moderada & $62,5(53,1-68,7)$ & \\
\hline & Boa & $62,5(56,2-71,8)$ & \\
\hline & Ótima & $67,1(57,8-82,8)$ & \\
\hline \multirow{4}{*}{ Índice de avaliação da QV } & Baixa & $75,0(59,3-75)$ & \multirow{4}{*}{0,389} \\
\hline & Moderada & $75,0(62,5-87,5)$ & \\
\hline & Boa & $75,0(75,0-75)$ & \\
\hline & Ótima & $75,0(75,0-87,5)$ & \\
\hline
\end{tabular}




\section{- DISCUSSÃO}

A atividade laboral do BM está condicionada a situações adversas e de alta periculosidade nas ocorrências durante o período de serviço, o que revela o grau de exigência e preparo para a execução desse tipo de trabalho ${ }^{(14)}$. Tal qual aqui evidenciado, trata-se de uma profissão exercida majoritariamente por homens com situação econômica estável, tanto em âmbito nacional ${ }^{(15)}$ quanto internacional ${ }^{(16-17)}$.

Com relação à faixa etária, foi possível notar que a maioria dos BM possui idade acima de 40 anos. O fato dos trabalhadores possuírem idade mais avançada pode proporcionar a diminuição da capacidade para o trabalho, ademais, esse tipo de situação demanda a incorporação de medidas de prevenção e cuidados em relação às atividades executadas de um trabalhador com esta idade ${ }^{(18)}$.

Observou-se no estudo que geralmente os BM que estão há mais tempo no serviço convivem com companheiro(a). A situação conjugal favorece a interação entre o bem estar emocional e o desenvolvimento com o trabalho, colaborando para uma melhor condição laboral ${ }^{(19)}$. Por conseguinte, considera-se a interação entre as condições laborais e a vida familiar um fator para uma melhor QV, reforçando a premissa de reflexão sobre a influência da família no aspecto psicológico do indivíduo e suas consequências na vida profissional ${ }^{(20)}$.

O bom preparo psicológico é de fundamental importância para o desenvolvimento laboral, pois o fato de vivenciarem situações de emergência, que exigem alto grau de comprometimento e ação rápida - como lidar com perdas de pessoas, não conseguir realizar o resgate em tempo hábil e estar exposto a eventos adversos - faz com que o profissional vivencie situações traumáticas. Tais fatos podem trazer consequências como o estresse pós-traumático, que compromete diretamente a saúde, além de afetar a QV do trabalhador ${ }^{(1,21)}$.

Por tais possibilidades de deterioração da saúde e por consequência da QV, é de suma importância o estudo da relação entre esta e as características laborais. Os BM com menos de cinco anos de serviço apresentaram melhor percepção de QV no domínio físico e relações sociais. Desta forma, nota-se que os indivíduos com menor tempo de trabalho nesta atividade provavelmente não apresentam comprometimento físico, tal fato pode decorrer devido estarem em início de carreira, além de não estarem expostos há tanto tempo aos fatores estressores. Complementarmente, outros estudos evidenciaram que trabalhadores que exercem a profissão há mais tempo sofrem com a condição física e jornada de trabalho, devido ao processo de desgaste e envelhecimento, o que pode comprometer a capacidade para o trabalho ${ }^{(2,22)}$.

Vale ressaltar que, com a realização do trabalho ao longo dos anos e com o processo fisiológico de envelhecimento, iniciam-se os problemas físicos e psíquicos, prejudicando o relacionamento interpessoal com os colegas de trabalho e familiares, potencializando o comprometimento da saúde e QV(22). Os indivíduos dos serviços de emergência estão sujeitos a vivenciar situações que comprometem a saúde e a QV por causa das condições relacionadas ao trabalho(23).

Ademais, o trabalho do profissional bombeiro é mediado por situações que oferecem riscos ocupacionais, físicos e ergonômicos. O fato de estar subindo escadas, retirar vítimas presas em ferragens após acidente, além do peso dos equipamentos, pode favorecer o desenvolvimento de problemas osteomusculares, assim, essas situações impossibilitam o trabalhador de exercer o seu labor, comprometendo a saúde, bem como a sua $\mathrm{QV}{ }^{(24)}$.

Os bombeiros com menos de cinco anos de serviço apresentaram melhor percepção de QV no domínio relações sociais, talvez pelo pouco tempo de experiência no trabalho, sendo necessário maior aporte e convívio com os colegas, no intuito de adquirir mais segurança e domínio na realização das atividades do serviço. O convívio social é um fator que possibilita maiores interações, diálogo na tomada das decisões, sendo um fator contribuinte para melhor QV(25).

Em consonância entre a QV e a capacidade para o trabalho, os resultados aqui postos demonstraram associação significativa entre as duas. Outros estudos revelam que tal associação pode decorrer do fato de a capacidade para o trabalho estar relacionada tanto com os fatores laborais quanto os fatores externos, além disso, poder associar-se diretamente com a percepção de QV ${ }^{(18,26)}$. 
Foi possível verificar que os BM com boa capacidade para o trabalho apresentaram melhor percepção de QV no domínio físico. Fatores relacionados à capacidade para o trabalho como o grau de satisfação com o serviço e a convivência são determinantes para que o profissional desempenhe sua função de maneira satisfatória ${ }^{(27)}$. No entanto, apesar da boa capacidade, não se exclui o fato de que as condições de trabalho associadas aos riscos físicos e ergonômicos podem comprometer a condição física, sendo que os fatores laborais como sobrecarga de trabalho e alternância de turnos interferem na qualidade do sono e alteram os hábitos do dia a dia do profissional, o que compromete a saúde e a QV (21-22).

Evidenciou-se que os bombeiros com ótima capacidade para o trabalho obtiveram uma melhor percepção sobre QV do domínio psicológico, enquanto os que possuíam uma baixa capacidade apresentaram o comprometimento na QV no referido domínio. Vale destacar que, por se tratar de uma profissão que lida diretamente com situações emergenciais, e por vivenciar momentos traumáticos, este tipo de trabalho pode desencadear reações psicológicas e mudanças no comportamento, assim como diminuir a capacidade para o trabalho ${ }^{(22,28)}$.

Considerando o domínio relações sociais com os aspectos da capacidade para o trabalho, houve diferença estatística, sugerindo que à medida que a capacidade melhora, a QV no domínio relações acompanha. Outros estudos revelam que, embora os grupos laborais possam apresentar relações harmônicas, os problemas emocionais e psicológicos podem surgir ao longo da profissão e afetar negativamente a boa convivência, bem como interferir no labor e na QV do individuo ${ }^{(7,29)}$.

No que se refere ao domínio meio ambiente ${ }^{(6)}$, observou-se tendência para maiores escores em relação a boa e ótima capacidade para o trabalho. Os indivíduos com baixa capacidade para o trabalho apresentaram a condição menos favorável à QV nessa dimensão. Alguns autores destacam que a falta de lazer associada com a falta de cuidados com a saúde, além dos problemas de ambiente do trabalho e o exercício de outras atividades laborais, podem comprometer o rendimento laboral e a saúde do bombeiro ${ }^{(24,26)}$.

Destaca-se que o tipo de desenho epidemiológico adotado permite avaliar um momento específico da saúde do indivíduo, não possibilitando a conclusão da causalidade entre as associações, o que impede a análise da temporalidade, o que se configura como uma limitação do estudo.

\section{CONCLUSÃO}

O estudo evidenciou que os BM com boa capacidade para o trabalho apresentaram melhor percepção de QV no domínio físico. Observou-se também que os BM com ótima capacidade para o trabalho apresentaram melhor percepção de QV nos domínios psicológico, relações sociais e meio ambiente. Os resultados reforçam que a atual capacidade para o trabalho do BM deve ser preservada. Os achados também poderão servir de parâmetro para realização de intervenções e melhorias no trabalho de profissionais de resgate como bombeiros, enfermeiros e médicos.

Embora os profissionais apresentem uma boa e ótima capacidade para o trabalho, é importante registrar que a capacidade em executar o trabalho sofre mudanças ao longo do tempo e problemas podem surgir no ambiente laboral, como aparecimento de riscos ocupacionais, problemas no relacionamento interpessoal com os colegas e estresse. Todos esses fatores influenciam na QV do indivíduo e na capacidade para o trabalho. Nessa perspectiva, espera-se que novos estudos possam ser efetuados acerca das populações de bombeiros no sentido de evidenciar os fatores que afetam a capacidade para o trabalho e a QV.

\section{REFERÊNCIAS}

1. Lima EP, Assunção AA, Barreto SM.Transtorno de Estresse Pós-Traumático (TEPT) em Bombeiros de Belo Horizonte, Brasil: Prevalência e Fatores Ocupacionais Associados. Psic:. Teor. e Pesq. [Internet]. 2015 [acesso em 2018 fev 24]; 31(2). Disponível em: http://dx.doi.org/10.1590/0102-37722015022234279288. 
3.Vidotti HGM, Coelho VHM, Bertoncello D, de Walsh IAP. Qualidade de vida e capacidade para o trabalho de bombeiros. Fisioter. Pesqui. [Internet]. 2015 [acesso em 2018 fev 18]; 22(3). Disponível: http://www.scielo.br/ scielo.php?pid=S1809-29502015000300231\&script=sci_abstract\&tlng=pt.

4. Lima C, Maia A, Ferreira R, Magalhães A, Nunes H, Pinheira S, et al. PTSD and quality of life among firefighters and municipal police forces. European Psychiatry. [Internet]. 2016 (Suppl 1) [acesso em 2018 fev 18]; 33. Disponível em: https://doi.org/10.1016/j.eurpsy.2016.01.1895.

5. The World Health Organization quality of life assessment (WHOQOL): development and general psychometric properties. Soc. Sci. Med. [Internet]. 1998 [acesso em 2015 jul]; 46(12). Disponível em: https://doi.org/10.1016/ S0277-9536(98)00009-4.

6. Fleck MPA, Louzada S, Xavier M, Chamovich E, Vieira G, Santos L,Pinzon V. Aplicação da versão em português do instrumento abreviado de avaliação da qualidade de vida "WHOQOL-Bref". Rev. Saúde Pública [Internet]. 2000) [acesso em 2015 jul 18]; 34(2). Disponível em: http://dx.doi.org/10.1590/S0034-89102000000200012.

7. Hilleshein EF, Lautert L. Capacidade para o trabalho, características sociodemográficas e laborais de enfermeiros de um hospital universitário. Rev. Latino-Am. Enfermagem [Internet]. 2012 [acesso em 2015 jul 18]; 20(3). Disponível em: http://dx.doi.org/10.1590/S0104-11692012000300013.

8. Smitha TD, Huhesa K, Dejoyb DM, Dyal MA. Assessment of relationships between work stress, work-family conflict, burnout and firefighter safety behavior outcomes.Safety Science. [Internet]. 2018 [acesso em 2018 fev 18]; 103. Disponível em: https://doi.org/10.1016/j.ssci.2017.12.005.

9. Ferreira RC, da Silveira AP, de SÁ MAB, Feres SBL, Souza JGS, Martis AMEBL. Transtorno mental e estressores no trabalho entre professores universitários da área da saúde. Trab. educ saúde. [Internet]. 2015 (Suppl 1)[acesso em 2015 ago 10];13. Disponível em: http://dx.doi.org/10.1590/1981-7746-sip00042.

10. Carpenter G, Carpenter T, Kimbrel N, Flynn E, Pennington M, Cammarata C, et al. Social support, stress and suicidal ideation in professional firefighters. Am J Health Behav. [Internet]. 2015 [acesso em 2018 fev 20]; 39(2). Disponível em: http://dx.doi.org/10.5993/AJHB.39.2.5.

11. Araújo EM, Costa MC, Hogan VK, Mota ELA, Araújo TM, Oliveira NF. Race/skin color differentials in potential years of life lost due to external causes. Rev. Saúde Pública. [Internet]. 2009 [acesso em 2015 ago 13]; 43(3). Disponível em: http://dx.doi.org/10.1590/S0034-89102009005000021.

12. Silva Junior SHA, Vasconcelos AGG, Griep RH, Rotenberg L.Validade e confiabilidade do índice de capacidade para o trabalho (ICT) em trabalhadores de enfermagem. Cad. Saúde Pública. [Internet]. 2011 [acesso em 2018 fev 20]; 27(6). Disponível em:http://dx.doi.org/10.1590/S0102-311X2011000600005.

13. Brasil. Ministério da Saúde. Resolução n. 466, de 12 de dezembro de 2012. Dispõe as diretrizes e normas regulamentadoras de pesquisas envolvendo seres humanos. Diário Oficial da União, Brasília, 13 jun. 2013. Seção 1, p. 59.

14. Oliveira AC, Machado BCA, Gama CS, Garbaccio JL, Damasceno QS. Biossegurança: conhecimento e adesão pelos profissionais do corpo de bombeiros militar de minas. Esc Anna Nery. [Internet]. 2013 [acesso em 2015 ago 14];17(1). Disponível em:http://dx.doi.org/10.1590/S1414-81452013000100020. 
15. Lima EP, Assunção AA, Barreto SM. Prevalência de depressão em bombeiros. Cad. Saúde Pública. [Internet]. 2015 [acesso em 2015 ago 17]; 31(4). Disponível em: http://dx.doi.org/10.1590/0102-311X00053414.

16. Davis J. Gallagher S. Physiological demand on firefighters crawling during a search exercise. International Journal of Industrial Ergonomics.[Internet]. 2014 [acesso em 2015 ago 17] ; 44(6). Disponível em: https://doi. org/10.1016/j.ergon.2014.10.001.

17. Mehta JP, Laverder SA, Hedman GE, Reichelt PA, Park S, Conrad KM. Evaluating the physical demands on firefighters using track-type stair descent devices to evacuate mobility-limited occupants from high-rise buildings. Applied. Ergonomics.[Internet]. 2015 [acesso em 2015 ago 18]; 45(3). Disponível em: https://doi.org/10.1016/j. apergo.2013.05.005.

18. Martin JIG, Duarte N, Gonçalves E, Cabral S, Silva C. Capacidade para o trabalho de bombeiros. Universitas: Ciências da Saúde. [Internet]. 2013 [acesso em 2018 fev 22]; 11(2). Disponível em: https://doi.org/10.5102/ucs. v11i2.2451.

19. Moura AL, dos Reis LM, Vannuchi MTO, Haddad MCL, Domansky RC. Capacidade para o trabalho de funcionários da prefeitura de um campus universitário público. Rev. Eletr. Enf. [Internet]. 2013 [acesso em 2018 fev 25]; 15(1). Disponível em: https://doi.org/10.5216/ree.v15i1.13574.

20. Martins FF, Lopes RMF, Farina M. Nível de estresse e principais estressores do motorista de transporte coletivo. Bol. - Acad. Paul. Psicol. [Internet]. 2014 [acesso em 2018 fev 26]; 34(87). Disponível em: http://pepsic. bvsalud.org/pdf/bapp/v34n87/a14.pdf.

21. Montero-Marin J, Prado-Abril J, Demarzo MMP, Gascon S., García-Campayo J. Coping with stress and types of burnout: explanatory power of different coping strategies. Plos. One. [Internet]. 2014 [acesso em 2018 fev 26]: 9(2) Disponível em:https://www.ncbi.nlm.nih.gov/pmc/articles/PMC3923838/.

22. Chen HC, Chou FH, Chen MC. A survey of quality of life and depression for Police officers in Kaohsiung, Taiwan.Qual Life Res. [Internet]. 2006 [ acesso em 2015 ago 21];15(5). Disponível em: http://dx.doi.org/ 10.1007/ s11136-005-4829-9.

23. Salvador RSP, Silva BASA, Lisboa MTL. Estresse da equipe de enfermagem do corpo de bombeiros no atendimento pré-hospitalar móvel. Esc Anna Nery. [Internet]. 2013 [acesso em 2015 ago 23];17(2). Disponível em: http://dx.doi.org/10.1590/S1414-81452013000200022.

24. Nunes DA, Fontana RT. Condições de trabalho e fatores de risco da atividade realizada pelo bombeiro. Cienc. Cuid. Saude. [Internet] 2012 [acesso em 2015 ago 25]; 11(4). Disponível em: http://dx.doi.org/10.4025/ cienccuidsaude.v11i4.18083.

25. Rueda FJM, Serenini ALP, Meireles E. Relação entre qualidade de vida no trabalho e confiança do empregado na organização. Rev. Psicol. Organ. Trab. [Internet]. 2014 [acesso em 2015 ago 25];14(3). Disponível em: http:// pepsic.bvsalud.org/pdf/rpot/v14n3/v14n3a06.pdf.

26. Milosevic M, Golubic R, Knezevic B, Golubic K, Bubas M, Mustajbegovic J. Work ability as a major determinant of clinical nurses' quality of life. J ClinNurs. [Internet]. 2011 [acesso em 2015 ago 25]; 20(19-20). Disponível em: http://dx.doi.org/10.1111/j.1365-2702.2011.03703.x. 
[acesso em 2018 fev 28];123. Disponível em: www.elsevier.com/locate/paid.

28. Shin H, Park YM, Ying JY, Kim B, Noh H, Lee SM. Relationships between coping strategies and burnout symptoms: a meta-analytic approach. Professional Psychology. Researchand. Practice. [Internet]. 2014 [acesso em 2018 mar 01]; 45(1). Disponível em: https://www.researchgate.net/publication/263936204.

29. Santos M, Almeida A. Principais riscos e fatores de risco ocupacionais associados aos bombeiros, eventuais doenças profissionais e medidas de proteção recomendadas. Rev. portuguesa de saúde ocupacional. [Internet]. 2016 [acesso em 02 mar 2018]; 1 Disponível em: http://www.rpso.pt/principais-riscos-e-fatores-derisco-ocupacionais-associados-aos-bombeiros-eventuais-doencas-profissionais-e-medidas-de-protecaorecomendadas/. 